\title{
Flexible Strain Sensor with Tunable Sensitivity via Microscale Electrical Breakdown in Graphene/Polyimide Thin Films
}

\author{
Yonggang Jiang, ${ }^{* \dagger}{ }^{\dagger}$ Qipei He,${ }^{\dagger}$ Jun Cai, ${ }^{\dagger}$ Dawei Shen ${ }^{\dagger}$ Xiaohe Hu, ${ }^{\dagger}$ and Deyuan Zhang ${ }^{\dagger, \S}$
}

$\dagger$ School of Mechanical Engineering and Automation, Beihang University, Beijing, 100191, China

$\S$ Beijing Advanced Innovation Center for Biomedical Engineering, Beihang University, Beijing, 100191, China

* Corresponding Author: jiangyg@buaa.edu.cn 


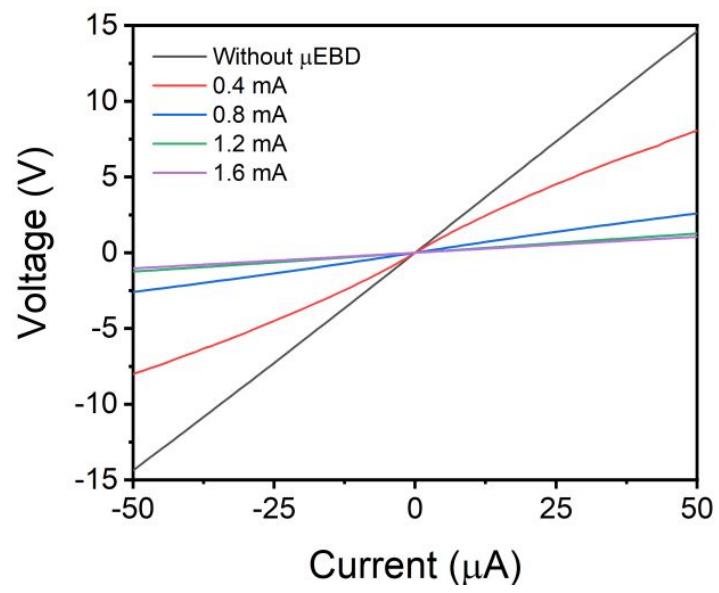

Figure S1. $V-I$ curves of G/PI sensors after $\mu \mathrm{EBD}$. 


\section{MAP 1}
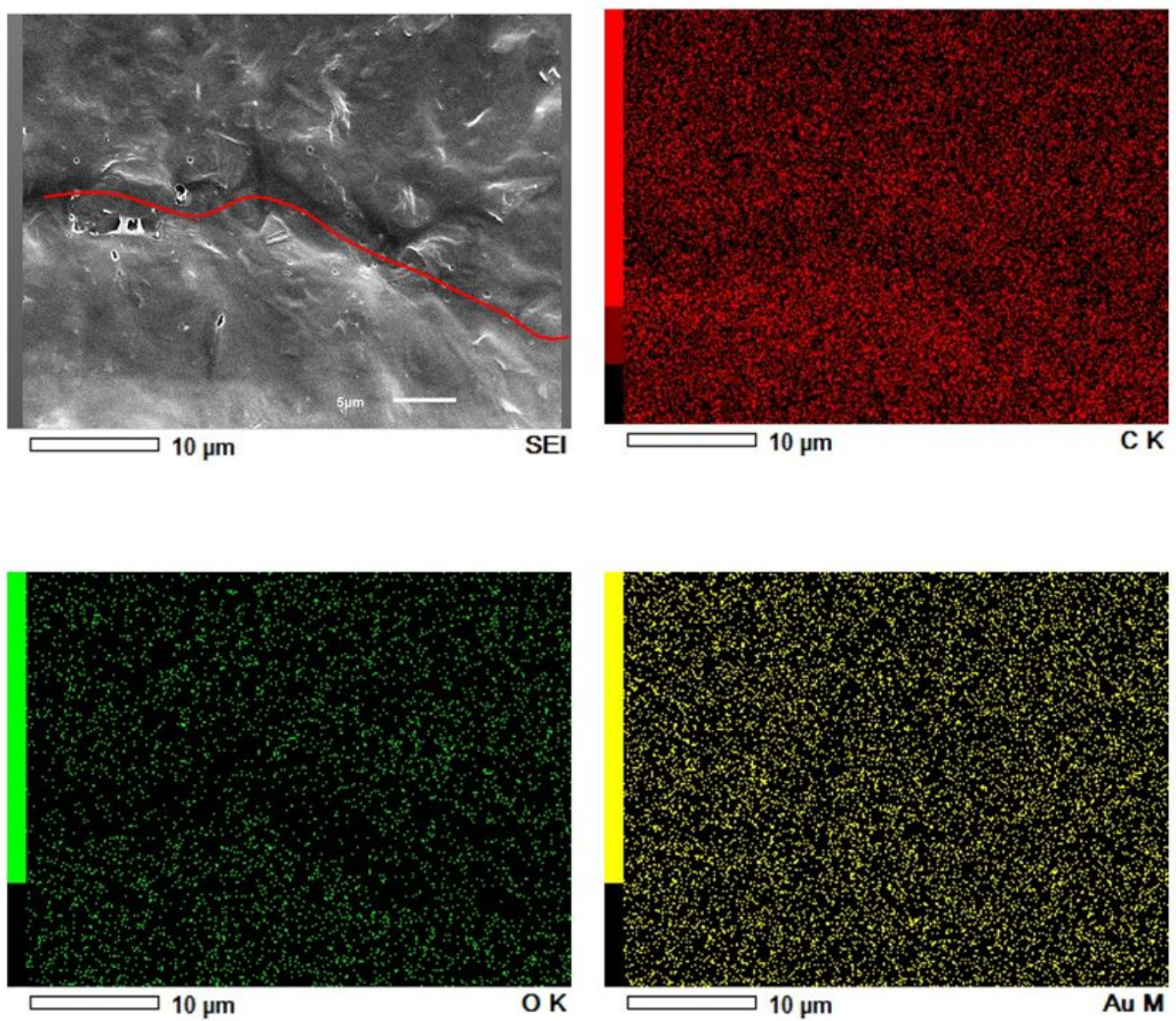

Figure S2. SEM images of the carbon trace and elemental mapping of the carbon trace. The red, green, and yellow points represent carbon, oxygen, and gold, respectively. 

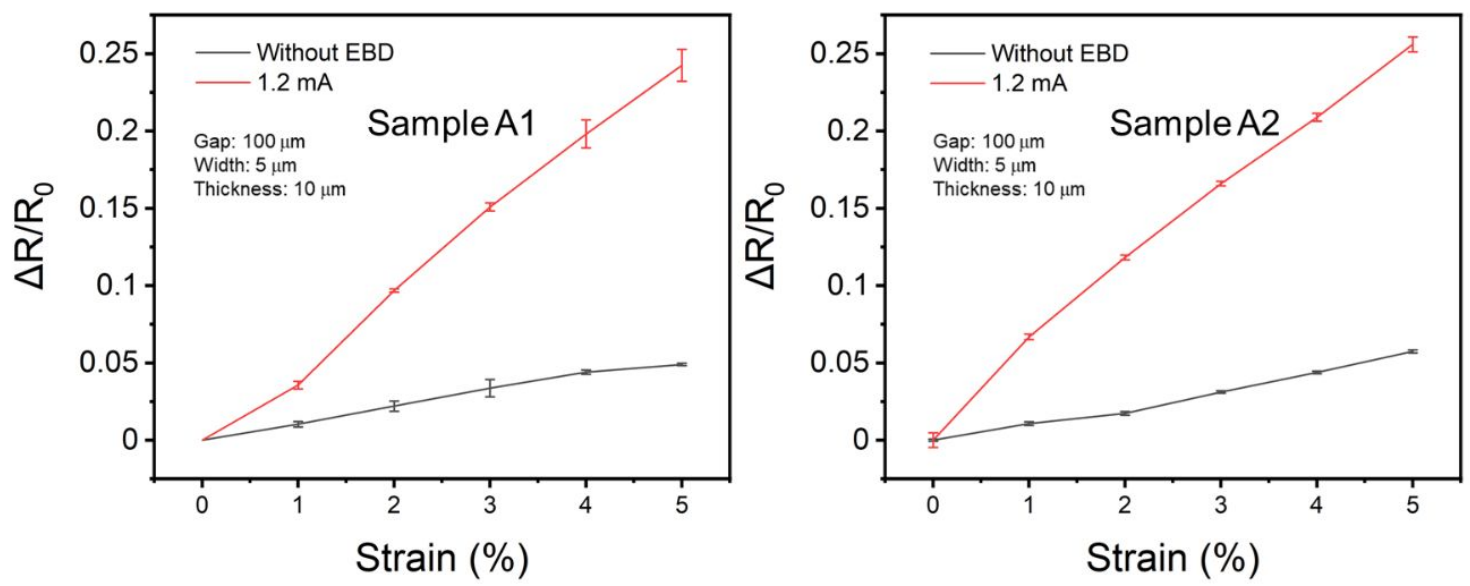

Figure S3. Relative resistance variation in response to the tensile strains of (a) Sample A1 and (b) Sample A2.
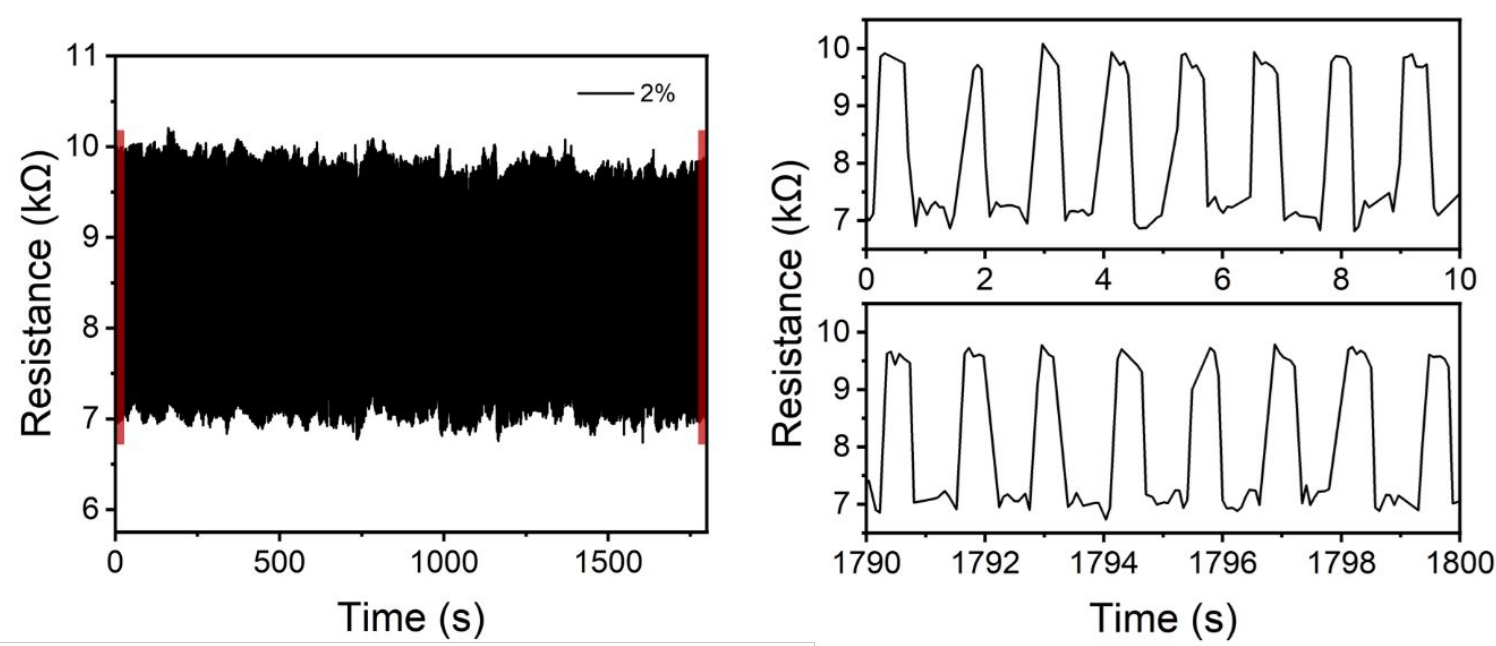

Figure S4. Real-time relative resistance-t curves of the sensor S2 fabricated at a $\mu$ EBD current of $0.8 \mathrm{~mA}$ for 1500 loading-release cycles. 


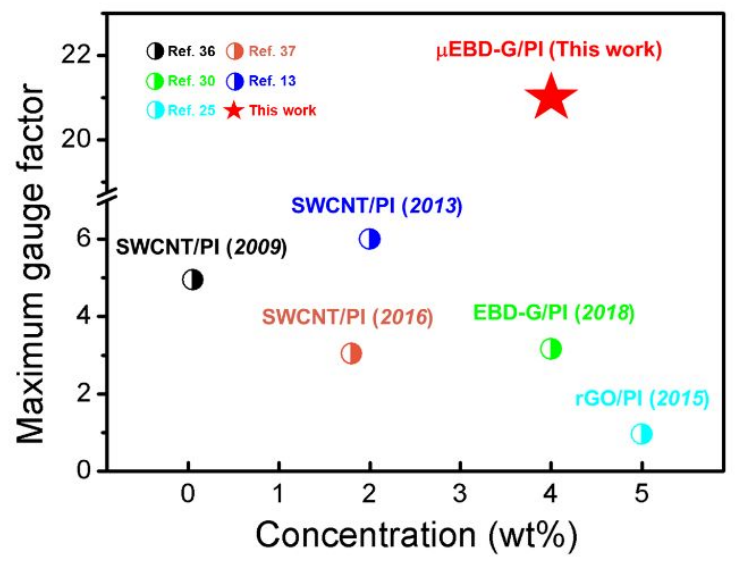

Figure S5. Comparison of the gauge factors of different carbon-based/polyimide sensors from literature.
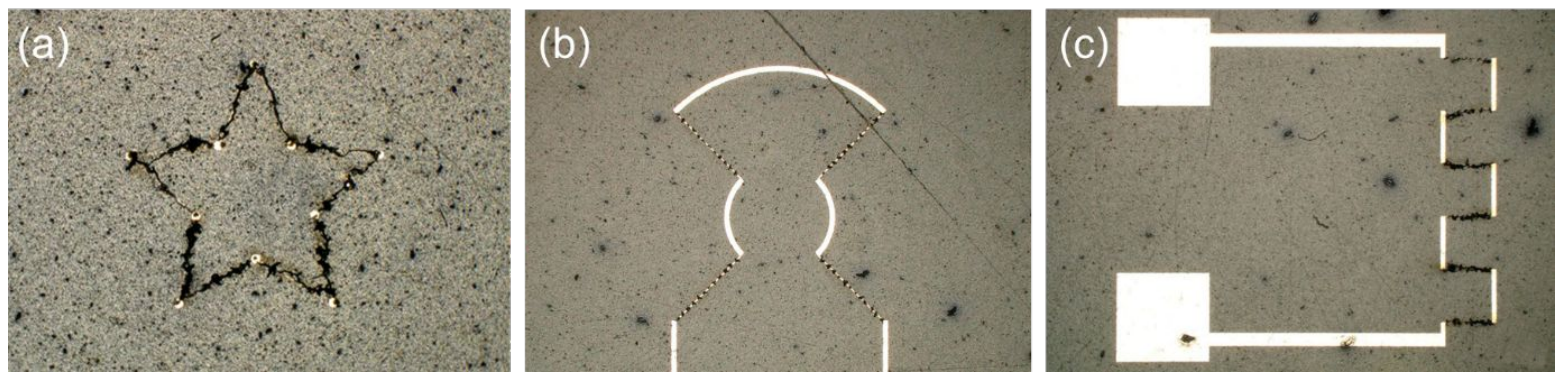

Figure S6. Photographs of the $\mu$ EBD-generated piezoresistive graphitic sensors with different patterns: (a) star, (b) fan, and (c) zigzag shapes. 


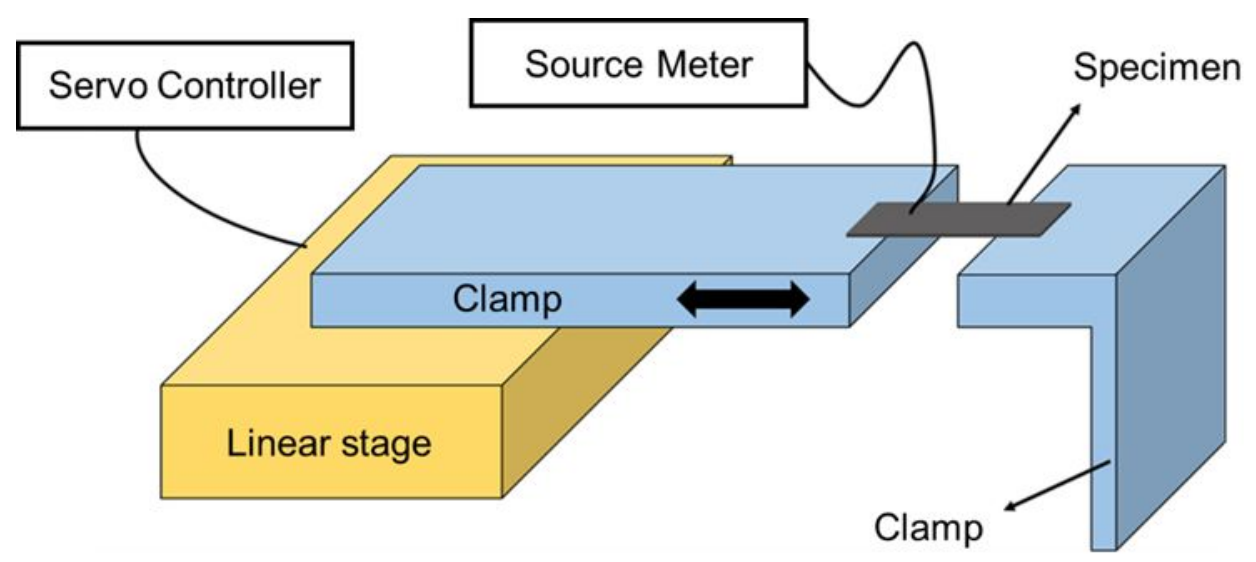

Figure S7. Schematic of the experimental setup for measuring the mechanical properties and obtaining piezoresistivity measurements.

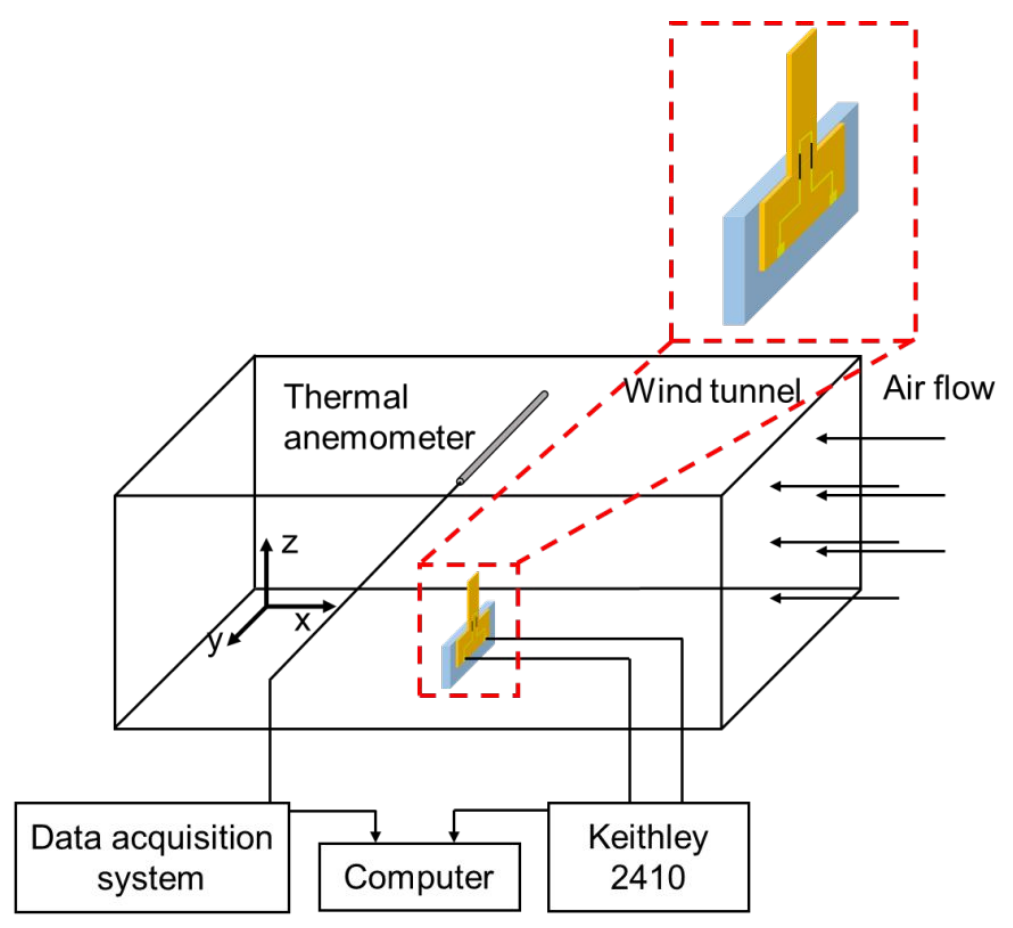

Figure S8. Schematic illustration of the sensor testing and wind tunnel experimental setup. 